\title{
Optimal Design of the Feeder-Bus Network Based on the Transfer System
}

\author{
Lianbo Deng, Wei Gao, Yanbing Fu, and Wenliang Zhou \\ School of Traffic and Transportation Engineering, Central South University, Changsha 410075, China \\ Correspondence should be addressed to Yanbing Fu; quanshuiqq@163.com
}

Received 25 August 2013; Accepted 25 October 2013

Academic Editor: Huimin Niu

Copyright (C) 2013 Lianbo Deng et al. This is an open access article distributed under the Creative Commons Attribution License, which permits unrestricted use, distribution, and reproduction in any medium, provided the original work is properly cited.

\begin{abstract}
This paper studied the classic feeder-bus network design problem (FBNDP), which can be described as follows: for the passenger travel demand between rail stations and bus stops on a given urban transit network, it designs the optimal feeder bus routes and frequencies so as to minimize the passengers' travel expense and the operator's cost. We extended the demand pattern of M-to- 1 in most existing researches to M-to-M. We comprehensively considered the passenger travel cost, which includes the waiting and riding cost on the bus, riding cost on rail, and transfer cost between these two transportation modes, and presented a new genetic algorithm that determines the optimal feeder-bus operating frequencies under strict constraint conditions. The numerical examples under different demand patterns have been experienced and analysed, which showed the robustness and efficiency of the presented algorithm. We also found that the distribution pattern of the travel demand has a significant influence on the feeder-bus network construction.
\end{abstract}

\section{Introduction}

As the two main transport modes in an urban transit system, the rail line usually plays the role of the transport trunk, while the feeder-bus network services act as a branch of and a supplement to the former. The integration and coordination of urban rail transit and the bus network can effectively promote the service efficiency and simultaneously improve the financial status of the system [1]. Stanger and Vuchic [2] pointed out that coordinative schedule optimization of the two modes could lead to operating cost savings. Some cities, such as Atlanta, Miami, and Washington, DC, gave top priority to the bus/rail coordination during the development process of the transportation systems. Dunn Jr. [3] showed that the coordination and integration of transport services have been a precondition for improving public transportation.

A good feeder-bus network significantly improves the public transport system's service level, operation efficiency, and market competitiveness. The feeder-bus network transports transfer passengers who come from the urban perimeter zone and will arrive at their final business or work destination by rail. Each bus line in the feeder-bus system usually connects to a special railway station and serves a sequence of bus stops with a certain frequency. Thus, the feeder-bus network design problem (FBNDP) can be described as follows: for a given urban rail line, the stop locations and the passenger travel demand between bus stops and railway stations, the optimal feeder bus routes, and their frequencies are determined so as to minimize the passenger travel cost and the bus operation cost [4-6].

\section{Literature Review}

The existing research on the FBNDP mainly follows two approaches, that is, the analytic approach and network programming (also known as mathematical programming). Most early research used analytic approaches to deduce the optimal route spacing, operating headway, and stop spacing based on assumptions regarding the shape of the street geometry and the spatial distribution of the passenger demand. According to the assumption of the early research, the demand is distributed in a rectangular region in which an existing rail line is serviced (accessed) by some parallel bus routes perpendicular to the rail line. Byrne and Vuchic [4] studied the optimal location and headway of parallel bus lines 
and presented a method to determine the optimal number of bus lines. On the basis of [4], Byrne [7] determined the lengths, positions, and headways of bus lines that could minimize the user travel time and operating costs in response to a general population density function with differing line speeds. Hurdle [8] studied the optimal location and schedule of parallel feeder lines with variable passenger density with different trip origins and times. Wirasinghe et al. [9] put forward optimization formulations for the optimal railway interstation spacing, feeder-bus zone boundary, and train headways mainly by the use of basic calculus in conjunction with continuum approximations of certain discrete parameters. Wirasinghe [10] researched a feeder-bus system with a demand pattern of M-to-1 (i.e., multiple bus stops and a single station). An approximate analytical model and corresponding solution algorithm were successfully applied to the Calgary (South Corridor) LRT system. Kuah and Perl [5] optimized the route spacing, operating headway, and stop spacing simultaneously, and analysed the influencing factors of bus stop spacing in three different cases. Supposing that the location of the rail line was predetermined, Chien and Schonfeld [11] cut the urban corridor into several traffic zones with different lengths but the same width and jointly optimized the rail line length, railway station spacing, bus headways, bus stop spacing, and bus route spacing under the conditions that the passenger flow density in each traffic zone was the same and that only one feeder-bus line connected to the same railway station. Chien and Yang [12] developed a model for finding the optimal bus route location and its operating headway in a heterogeneous service area while considering intersection delays. In these models, irregular and discrete M-to-1 demand distributions were considered. A heuristic algorithm [12] and later a genetic algorithm [13] were designed to solve the above model.

In recent decades, the network programming approach has been introduced to deal with the FBNDP. In this approach, the urban transport network is usually represented by a graphic framework, in which nodes denote bus stops or railway stations and links denote route segments between the two successive nodes. For simplicity, it takes bus stops as the origin and the rail station as the destination of the travel demand. Kuah and Perl [6] developed a mathematical programming model for the FBNDP under the M-to-1 demand pattern and designed a heuristic algorithm based on the savings approach. The demand pattern of $\mathrm{M}$-to-M (i.e., the demand pattern in which multiple stations are the destinations) was transformed and generalized to M-to-1 by separating the bus stops into dummy child nodes with the same number of railway stations. Furthermore, the sensitivity of the model was analysed for changes in the design objective, passenger demand variability, vehicle capacity, labour and fuel costs, and rail line. Martins and Pato [14] further presented two strategies to generate the initial solution (i.e., the continuous construction method and the two-phase method) and designed a local search as well as tabu search heuristics with diversification and intensification strategies. Shrivastav and Dhingra [15] discussed the FBNDP for the operational integration of the suburban railway and bus transit system and developed a heuristic algorithm using different node selection and insertion strategies. Kuan et al. mainly focused on the application of metaheuristic algorithms to the FBNDP, such as simulated annealing and tabu search [16], genetic algorithm, and ant colony optimization [17] and analysed and compared the optimal results obtained by these algorithms.

More recently, Ciaffi et al. [18] dealt with the FBNDP using a two-phase method. In the first phase, a heuristic algorithm was used to generate two different and complementary sets of feasible routes, in order to provide a proper balance between the maximization of the service coverage area and the minimization of the overall travel time. In the second phase, the sets generated in the first phase were used as input data and a GA was designed to find a suboptimal set of routes with the associated frequencies.

Almost all the existing research assumed that the travel demand starts from multiple bus stops but ends at one particular railway station near the central business district (i.e., the demand pattern of M-to-1). In this paper, this drawback is modified to adapt well to the realistic passenger distribution. We consider that origin-destination (OD) pairs may exist between any bus stop and any railway station (M-to-M). After computing the passenger travel cost from the view of the whole feeder-bus network, we present a network optimization model with the objective of minimizing the passenger travel cost and the bus operation cost. Furthermore, a new generation algorithm (GA) is developed and the optimal results under different passenger patterns are analysed and compared.

\section{Problem Description}

The feeder-bus network mainly transports transfer passengers between the bus and the railway system. We regard the nodes, including bus stops and rail stations, as traffic points of passenger collection and distribution. In the classical FBNDP, all passengers are supposed to have a certain railway station as their destination. We widen this assumption to the M-to-M pattern; that is, the passenger demand is distributed between any bus stop and any railway station. Under this demand pattern, the feeder-bus routes obey the following assumptions in most previous studies.

(1) Each bus stop is served by one feeder-bus route only.

(2) Each bus route does not cross its feeder railway station but terminates at the station.

(3) All bus routes have a uniform capacity and operating speed, and the volume of transport passengers should not exceed their capacity.

(4) Each bus must halt at all the stops along its route and the skip-stop running strategy is not considered.

When the feeder-bus network obeys the above assumptions, there is an M-to- 1 connection relationship between bus stops and railway stations. However, the route structure and the feeder station are influenced by the demand distribution of the M-to-M demand pattern, and the operating frequency can be obtained through the optimal cost of the transit operator and transfer passengers. 
Considering an urban public transit network composed of $I$ bus stops and $J$ railway stations, we denote the set of bus stops by $B=\{1, \ldots, I\}$, the set of railway stations by $T=\{I+$ $1, \ldots, I+J\}$, and the set of network nodes by $N=B \cup T$. We also let the distance of a section between two adjacent nodes $i, j$ be $L_{i j}, i, j \in N$, the bus operating speed $v_{B}$, and the train speed $v_{T}$.

Due to the time fluctuation of the urban passenger demand, we can determine the bus schedule for each planning period (such as one hour in the morning peak). In the given period, the demand between $i$ and $d$ can be expressed as $P_{i d}$, for $i \in B, d \in T$.

Supposing the feeder-bus network $\Omega$ consists of $K$ feeder-bus routes, the path structure of bus route $k(k=$ $1, \ldots, K)$ is denoted by $\omega_{k}=\left\{n_{1}^{k}, n_{2}^{k} \ldots, n_{P_{k}-1}^{k}, n_{P_{k}}\right\}$, in which $n_{P_{k}} \in T$ is the feeder railway station and $n_{1}^{k}, n_{2}^{k}, \ldots, n_{P_{k}-1}^{k} \in B$ are bus stops along the route. The operating frequency of route $k$ is denoted by $f_{k}$.

\section{Model Construction}

The constraints of the feeder-bus network can be obtained according to the above assumptions and the transit operating requirement. Compared with the demand pattern of M-to1 , the network construction constraints under the M-to-M pattern are completely uniform. However, the generalized travel cost of transfer passengers under the demand pattern of $\mathrm{M}$-to-M will have a more complex influence on their choice of feeder station in the railway line, thus affecting the feederbus route structure and further the network construction.

4.1. Constraints Analysis. To represent the feeder-bus network constraints, $Y_{i j}$ and $X_{i h k}$ are defined to denote the relationship between nodes and routes:

$$
Y_{i j}= \begin{cases}1, & \text { if bus node } i \text { is assigned to rail node } j \\ 0, & \text { otherwise, }\end{cases}
$$$$
\forall i=1, \ldots, I ; j=I+1, \ldots, I+J,
$$

$$
X_{i h k}= \begin{cases}1, & \text { if node } i \text { precedes node } h \text { on bus route } k \\ 0, & \text { otherwise, }\end{cases}
$$

$$
\forall i, h=1, \ldots, I+J ; k=1, \ldots, K .
$$

All the constraints that need to be satisfied by the feederbus network are as follows.

(1) Connectedness constraint of the feeder-bus network: in the feeder-bus network, any subset of bus stops must link to feeder stations directly or via other bus stops, that is, the following connectedness constraint:

$$
\sum_{i \notin H} \sum_{h \in H} \sum_{k=1}^{K} X_{i h k} \geq 1, \quad \forall H,
$$

where $H$ is a set containing all the rail stations and some bus stops. Obviously, it is also a subset of $N$.
(2) Integrity constraints of the feeder-bus route: each bus route must link to a single railway station:

$$
\sum_{i=1}^{I} \sum_{j=I+1}^{I+I} X_{i j k}=1, \quad k=1, \ldots, K
$$

A route terminates at a certain feeder station $d \in T$ to which the route passengers are transported,

$$
\sum_{i=I+1}^{I+J} \sum_{j=1}^{I+I} X_{i j k}=0, \quad k=1, \ldots, K .
$$

In addition, a feeder-bus route should include at least one stop and one feeder station, that is, the following nonempty constraint:

$$
\sum_{i=1}^{I} \sum_{j=1}^{I+I} X_{i j k} \geq 1, \quad k=1, \ldots, K .
$$

(3) Relationship constraints between routes and nodes:

feeder-bus route $k$ must halt at stop $i$ only once, $i$ should be served only by route $k$ when $i \in \omega_{k}$, and route $k$ should be acyclic:

$$
\begin{gathered}
\sum_{k=1}^{K} \sum_{h=1}^{I+J} X_{i h k}=1, \quad i=1, \ldots, I, \\
\sum_{k=1}^{K} \sum_{p=1}^{I} X_{p i k} \leq 1, \quad i=1, \ldots, I, \\
\sum_{h=1}^{I+J} X_{i h k}-\sum_{p=1}^{I} X_{p i k} \geq 0, \quad i=1, \ldots, I ; k=1, \ldots, K .
\end{gathered}
$$

In addition, relationship variable $Y_{i j}$ between route $k$ and nodes $i, j$ satisfies the following constraint:

$$
\begin{array}{r}
\sum_{h=1}^{I+J} X_{i h k}+\sum_{p=1}^{I} X_{p j k}-Y_{i j} \leq 1, \quad i=1, \ldots, I ; \\
j=I+1, \ldots, I+J ; \quad k=1, \ldots, K .
\end{array}
$$

(4) Capacity constraints of the feeder-bus route and network:

for route $k$, operating frequency $f_{k}$ should meet the passenger transport capacity; that is,

$$
\sum_{i=1}^{I} \sum_{d=I+1}^{I+J} P_{i d} \sum_{h=1}^{I+J} X_{i h k} \leq f_{k} C \rho, \quad k=1, \ldots, K,
$$

where $C$ is the bus capacity and $\rho$ is the load factor. 
Meanwhile, the route operating frequencies are restricted by the total running mileage of bus vehicles in the planning period; that is,

$$
\sum_{k=1}^{K} f_{k} \sum_{i=1}^{I} \sum_{h=1}^{I+J} L_{i h} X_{i h k} \leq \frac{1}{2} N v_{B}(T-t)
$$

where $t$ is the average servicing and turn-around time of every bus vehicle in period $T$ and $N$ is the number of vehicles that can be used in $T$.

4.2. Cost Analysis. In order to ensure the good market competitiveness and operation effect, the feeder-bus network needs to consider the benefits both of passengers and of operators. For passengers, this refers to their generalized travel cost, which can be divided into three parts: the waiting and riding cost on the bus, the riding cost on the rail line, and the transfer cost between these two modes. Compared with $[6,14]$, the transfer cost is taken into account in this paper and the contents of the passenger travel cost are closer to a realistic situation.

In the passenger travel cost, the bus waiting and bus riding costs are $\lambda_{w} \sum_{k=1}^{K}\left(1 / 2 f_{k}\right) \sum_{d=I+1}^{I+J} \sum_{i=1}^{I} \sum_{h=1}^{I+J} P_{i d} X_{i h k}$ and $\sum_{k=1}^{K}\left\{L_{k} \sum_{d=I+1}^{I+J} P_{n_{1}^{k} d}+\left(L_{k}-L_{n_{1}^{k} n_{2}^{k}}\right) \sum_{d=I+1}^{I+J} P_{n_{2}^{k} d}+\cdots+\right.$ $\left.L_{n_{P_{k}-1}^{k} n_{P_{k}}} \sum_{d=I+1}^{I+J} P_{n_{P_{k}-1}^{k} d}\right\} \lambda_{r} / \nu_{B}$, respectively, where $\lambda_{w}$ and $\lambda_{r}$ are the corresponding monetary cost conversion coefficients, $P_{k}$ and $L_{k}$ are the total passenger demand and length of route $k$; the transfer cost at the feeder station is closely related to the transfer facilities and train operating density, so the transfer cost for every passenger at station $j(j=I+$ $1, \ldots, I+J)$ can be expressed as $\lambda_{j}$, and the total rail riding cost is $\left(\lambda_{T} / v_{T}\right)\left(\sum_{d=I+1}^{I+J} \sum_{j=I+1}^{I+J} \sum_{i=1}^{I} P_{i d} Y_{i j} L_{j d}\right)$, where $\lambda_{T}$ is the corresponding monetary cost conversion coefficient.

For the bus operator, the operating cost of feeder-bus routes mainly relates to operating mileages and frequencies, which are denoted as $2 \lambda_{b} \sum_{k=1}^{K} f_{k} \sum_{h=1}^{I+J} \sum_{i=1}^{I} L_{i h} X_{i h k}$, where $\lambda_{b}$ is the cost per carriage per mile.

4.3. Optimization Model. Considering the benefits of both the passengers and the operator, the objective function of feeder-bus network programming is to minimize the passengers' generalized travel cost and the operator's cost:

$\min Z$

$$
\begin{aligned}
= & \frac{\lambda_{T}}{v_{T}}\left(\sum_{d=I+1}^{I+J} \sum_{j=I+1}^{I+J} \sum_{i=1}^{I} P_{i d} Y_{i j} L_{j d}\right) \\
& +\lambda_{w} \sum_{k=1}^{K} \frac{1}{2 f_{k}} \sum_{d=I+1}^{I+J} \sum_{i=1}^{I} \sum_{h=1}^{I+J} P_{i d} X_{i h k} \\
& +\left(\sum _ { k = 1 } ^ { K } \left\{L_{k} \sum_{d=I+1}^{I+J} P_{n_{1}^{k} d}+\left(L_{k}-L_{n_{1}^{k} n_{2}^{k}}\right) \sum_{d=I+1}^{I+J} P_{n_{2}^{k} d}\right.\right.
\end{aligned}
$$

$$
\begin{gathered}
\left.\left.+\cdots+L_{n_{P_{k}-1}^{k} n_{P_{k}}} \sum_{d=I+1}^{I+J} P_{n_{P_{k}-1}^{k}} d\right\} \lambda_{r}\right) \times\left(v_{B}\right)^{-1} \\
+\sum_{j=I+1}^{I+J} \lambda_{j}\left(\sum_{d=I+1}^{I+J} \sum_{i=1}^{I} P_{i d} Y_{i j}-P_{i j}\right) \\
+2 \lambda_{b} \sum_{k=1}^{K} f_{k} \sum_{h=1}^{I+J} \sum_{i=1}^{I} L_{i h} X_{i h k} .
\end{gathered}
$$

Objective function (10) and constraints (2) (9) constitute the optimization model of the FBNDP. The optimization model has the following main characteristics.

(1) The transfer cost is included in the passenger travel cost, so the travel cost on the transfer network can be calculated overall.

(2) The optimal objective is to minimize the passenger travel cost and transit operating cost, so benefits to users and operators in the public transit system are both considered.

(3) The demands are not limited to a single destination (M-to-1) and are extended to the distribution between every origin and destination (M-to-M), so the model accords well with a realistic demand distribution.

\section{Model Solution}

The optimization model of the feeder-bus network, with many 0-1 variables and many constraints, is NP hard [14]. It is essentially a route optimization problem in the field of operations research, which is appropriate for solving by some intelligent heuristic algorithms. Thus, in this paper, we present a genetic algorithm for the model. In the generation process of initial solution individuals and new population individuals, we use the following strategy for constructing routes: it firstly generates the connection relations of stops and stations, then finally optimizes the routing path structure and determines the operating frequency. In the process of generating feeder-bus routes, feeder relations and the path of each bus route are determined according to the cost of candidate routes.

5.1. Optimal Frequency for Each Route. In feeder-bus network $\Omega$, the path structure and cost of a feeder-bus route are not correlated with the other routes; therefore, the operating frequency of each route can be set independently. For feederbus route $\omega_{k}$, according to the objective function (10), its cost is

$C_{k}$

$$
=\frac{\lambda_{T}}{v_{T}}\left(\sum_{i=n_{1}^{k}}^{n_{P_{k}-1}^{k}} \sum_{d=I+1}^{I+J} P_{i d} L_{n_{P_{k}}} d\right)+\frac{\lambda_{w} P_{k}}{2 f_{k}}
$$




$$
\begin{aligned}
& +\lambda_{n_{P_{k}}}\left(P_{k}-\sum_{i=n_{1}^{k}}^{n_{P_{k^{-}}}^{k}} P_{i n_{P_{k}}}\right)+2 \lambda_{b} f_{k} L_{k} \\
& +\left(\lambda _ { r } \left\{L_{k} \sum_{d=I+1}^{I+J} P_{n_{1}^{k} d}+\left(L_{k}-L_{n_{1}^{k} n_{2}^{k}}\right)\right.\right. \\
& \left.\left.\quad \times \sum_{d=I+1}^{I+J} P_{n_{2}^{k} d}+\cdots+L_{n_{P_{k}-1}^{k} n_{P_{k}}} \sum_{d=I+1}^{I+J} P_{n_{P_{k}-1}^{k}} d\right\}\right) \times\left(v_{B}\right)^{-1} .
\end{aligned}
$$

In order to minimize $C_{k}$, the optimal operating frequency without any constraint can be obtained by the first-order optimality condition of $C_{k}$ with respect to $f_{k}$ in (11) as follows:

$$
f_{k}^{*}=\frac{1}{2} \sqrt{\frac{\lambda_{w} P_{k}}{\lambda_{b} L_{k}}}
$$

Then, the optimal operating frequency of $\omega_{k}$ under constraint (8) is

$$
\bar{f}_{k}^{*}=\max \left\{\frac{1}{2} \sqrt{\frac{\lambda_{w} P_{k}}{\lambda_{b} L_{k}}}, \frac{P_{k}}{(C \rho)}\right\} .
$$

Under the optimal frequency $\bar{f}_{k}^{*}$, the minimum route cost of $\omega_{k}$ is

$$
\begin{aligned}
& C_{k}^{*} \\
& =\frac{\lambda_{T}}{v_{T}}\left(\sum_{i=n_{1}^{k}}^{n_{P_{k}-1}^{k}} \sum_{d=I+1}^{I+J} P_{i d} L_{n_{P_{k}} d}\right)+\frac{\lambda_{w} P_{k}}{2 \bar{f}_{k}^{*}} \\
& +\lambda_{n_{P_{k}}}\left(P_{k}-\sum_{i=n_{1}^{k}}^{n_{P_{k}-1}^{k}} P_{i P_{P_{k}}}\right)+2 \lambda_{b} \bar{f}_{k}^{*} L_{k} \\
& +\left(\lambda _ { r } \left\{\sum_{k} \sum_{d=I+1}^{I+J} P_{n_{1}^{k} d}+\left(L_{k}-L_{n_{1}^{k} n_{2}^{k}}\right)\right.\right. \\
& \left.\left.\quad \times \sum_{d=I+1}^{I+J} P_{n_{2}^{k} d}+\cdots+L_{n_{P_{k}-1}^{k} n_{P_{k}}} \sum_{d=I+1}^{I+J} P_{n_{P_{k}-1}^{k} d} d\right\}\right) \times\left(v_{B}\right)^{-1} .
\end{aligned}
$$

Thus, the total operating cost of $\Omega$ is

$$
Z(\Omega)=\sum_{k=1}^{K} C_{k}^{*}
$$

Particularly, the optimal operating frequency $\bar{f}_{i j}^{*}$ of directlink route $\omega_{i j}$, which directly links stop $i \in B$ and station $j \in T$, is $\max \left\{(1 / 2) \sqrt{\lambda_{w} \sum_{d=I+1}^{I+J} P_{i d} / \lambda_{b} L_{i j}}, \sum_{d=I+1}^{I+J} P_{i d} /(C \rho)\right\}$. Then, accordingly, the total operating cost $D C_{i j}$ of $\omega_{i j}$ is

$$
\begin{aligned}
D C_{i j}= & \frac{\lambda_{T}}{v_{T}}\left(\sum_{d=I+1}^{I+J} P_{i d} L_{j d}\right)+\frac{\lambda_{w} \sum_{d=I+1}^{I+J} P_{i d}}{2 \bar{f}_{i j}^{*}} \\
& +\frac{\lambda_{r} L_{i j} \sum_{d=I+1}^{I+J} P_{i d}}{v_{B}}+\lambda_{j}\left(\sum_{d=I+1}^{I+J} P_{i d}-P_{i j}\right)+2 \lambda_{b} \bar{f}_{i j}^{*} L_{i j} .
\end{aligned}
$$

5.2. Optimization Strategy for the Route Structure. In the process of the GA, all routes $\omega_{k}$ in the population individuals need to optimize the path structures when the nodes in the routes are changed or the generation is updated. When the stops served by route $\omega_{k}$ and the end (feeder station) of $\omega_{k}$ are determined, the path structure optimization of $\omega_{k}$ can come down to an open vehicle routing problem with one depot (i.e., the feeder station). Thus, we adopt $2 p_{k}$ iterations of the 2-opt strategy to optimize the route structure, where $p_{k}$ is the number of nodes on the route. Because of the high probability that some elite gene segments of the offspring can be inherited from the parents, the fixed iterations of the route structure optimization for every individual of each generation can improve the population quality.

5.3. Genetic Coding. In this paper, we use an intuitional style to code the feeder-bus plan. Thus, every node in the network is expressed by a natural number; we also identify the bus stops or railway stations with different number sets. Then, a feeder-bus route would be a number substring ending at a railway station, and the whole coding scheme of the network would be the sequential connection of these route substrings. Note that the coding length of the network plan depends on the total route number designed in the transit system and it is not fixed.

For example, when $B=\{1,2,3,4,5,6,7,8,9,10\}$ and $T=$ $\{11,12,13\}$, a sample feeder-bus network can be expressed as 12311461257128910 13; substrings $12311,4612,57$ 12, and 891013 stand for 4 feeder-bus routes, respectively, in which the boldface numbers stand for the feeder stations.

Based on objective function (10) and considering the feasibility of constraint (9) simultaneously, the fitness function of individual $\Omega$ is constructed as follows:

$$
F(\Omega)=\frac{1}{\lambda Z(\Omega)}+\lambda\left[\frac{1}{2} N v_{B}(T-t)-\sum_{k=1}^{K} f_{k} \sum_{i=1}^{I} \sum_{h=1}^{I+J} L_{i h} X_{i h k}\right],
$$

where $\lambda$ is a penalty factor.

5.4. Initial Population. Each feeder-bus network in the initial population is generated one route by one route. Due to that, all the routes end at railway stations, so a feeder-bus station $j \in T$ can be selected first; then we choose a bus stop $i \in B$ with a choice probability, insert this stop into an existing route that terminates at node $j$, or link it directly with node $j$ to 
generate a new route. In this way, the generation of a network is finished when all the bus stops have been selected.

In order to improve the individual quality of the initial population, we construct a function to evaluate the connecting relationship between a bus stop and a railway station so that the roulette selection method is utilized to compute the selection probability. Let $D C_{i j}^{*}=\max _{i \in B} D C_{i j}+\min _{i \in B} D C_{i j}$; then, the evaluation function between stop $i$ and station $j$ is

$$
F_{i j}=D C_{i j}^{*}-D C_{i j}
$$

For station $j$, the selection probability of stop $i$ is $F_{i j} / \sum_{i=1}^{I} F_{i j}$.

The construction algorithm of a feeder-bus network of the initial population is as follows.

Algorithm 1. (1) Let $B^{\prime}=B$, where $B^{\prime}$ stands for the set of stops that could be selected to construct the current feederbus route. $\Omega=\emptyset$ is a feeder-bus network and $k=0$ is the number of routes that have been generated.

(2) If $B^{\prime}=\phi$, the algorithm terminates. Otherwise, randomly select a station $j$ from $T$ with equal probability.

(3) A random number $R \in(0,1)$ is created according to uniform distribution. Take a stop $i$ from $B^{\prime}$, which satisfies $\sum_{i=1}^{i-1} F_{i j} / \sum_{i=1}^{I} F_{i j} \leq R<\sum_{i=1}^{i} F_{i j} / \sum_{i=1}^{I} F_{i j}$.

(4) Let $\omega_{i j}$ be the direct route from $i$ to $j$ and $M$ the number of routes ending at $j$ in $\Omega$. If $M=0$, set $\Omega=\Omega \cup$ $\left\{\omega_{i j}\right\}, B^{\prime}=B^{\prime} \backslash i, k=k+1$; go to (2). Otherwise, for $m=$ $1, \ldots, M$, insert $i$ into the $m$ th route that ends at $j$ in $\Omega$, form the corresponding network $\Omega_{m}$ and let $\Omega_{M+1}=\Omega \cup$ $\left\{\omega_{i j}\right\}$; let $\Omega^{\prime}=\arg \min \left\{Z\left(\Omega_{m}\right) \mid m=1, \ldots, M+1\right\}$; if $\Omega^{\prime}=$ $\Omega_{M+1}, k=k+1 ; \Omega=\Omega^{\prime}, B^{\prime}=B^{\prime} \backslash i$; go to (2).

Let $n$ be the size of the initial population, which could be reasonably determined according to the values of $I$ and $J$. The initial population is constructed by calling Algorithm $1 n$ times.

\subsection{Genetic Operators}

(1) Selection and Replication Operator. To strengthen the searching ability of the GA, competition and intrusion mechanisms are introduced to construct the parent population. The former is that the population with $n$ individuals is replicated to form a new population with $2 n$ individuals firstly; then these $2 n$ individuals are divided into $n$ pairs arbitrarily and the better individuals are preserved by comparing the fitness of each pair. The latter means that $\alpha n$ new individuals by Algorithm 1 are introduced to replace the $\alpha n$ worst ones of the current population, where $\alpha$ is an intrusion ratio. The value of $\alpha$ is dynamically controlled in the range of $[\underline{\alpha}, \bar{\alpha}]$. Set $\alpha=\min (2 * \alpha, \bar{\alpha})$ when the best solution has not been improved in $T_{\alpha}$ times generations; set $\alpha=\underline{\alpha}$ when the best solution is improved.

(2) Crossover Operator. Here, we take two parents to generate two offspring with crossover probability $P_{c}$. In order to ensure that offspring individuals can inherit the elite gene from the parents, gene segments of routes of which the average cost per passenger is lower are chosen and inserted into the offspring individuals. In individual $\Omega_{l}$, let the node of locus $i$ be $V_{l}^{i}$, let the number of routes $n_{1}$, and let the average cost per passenger of $\omega_{k}$ be $A C_{k}$.

Choose the two parents $\Omega_{l_{1}}$ and $\Omega_{l_{2}}$ and generate offspring $\Omega_{l}$ based on $\Omega_{l_{1}}$. Firstly, compare the average travel cost per passenger of the two gene segments that start the node $V_{l_{1}}^{1}$, the first locus of $\Omega_{l_{1}}$, in $\Omega_{l_{1}}$ and $\Omega_{l_{2}}$. The better option is to choose $\Omega_{l}$ and delete $V_{l_{1}}^{1}$ from the two parents. Then, make the comparison of the gene segments that start the last node in $\Omega_{l}$ in the two parents until $\Omega_{l}$ terminates at a railway station and a route in $\Omega_{l}$ is generated. Then, delete the routes that cannot satisfy constraint (5) or combine the two shortest routes in each parent. With the above method, gene segments of the parents are selected to join the offspring constantly, and the routes of the offspring are constructed one by one until the whole offspring feeder-bus network is formed. The detailed algorithm is described as follows.

Algorithm 2. (1) Take the parents $\Omega_{l_{1}}$ and $\Omega_{l_{2}}$, and the generated offspring $\Omega_{l}$. Set $\Omega_{l_{1}}^{\prime}=\Omega_{l_{1}}, \Omega_{l_{2}}^{\prime}=\Omega_{l_{2}}$, and $i=1$.

(2) If $n_{l_{1}}^{\prime}=0$, go to (7); otherwise, select the locus $i^{\prime}=$ 1 and the corresponding route is $\omega_{k_{1}}$ in $\Omega_{l_{1}}^{\prime}$. Set $V_{l}^{i}=V_{l_{1}}^{i^{\prime}}$.

(3) Find locus $j^{\prime}$ from $\Omega_{l_{2}}^{\prime}$, satisfying $V_{l_{2}}^{j^{\prime}}=V_{l_{1}}^{i^{\prime}}$, and the corresponding route is $\omega_{k_{2}}$; go to (5).

(4) Find locus $i^{\prime}$ from $\Omega_{l_{1}}^{\prime}$, satisfying $V_{l_{2}}^{j^{\prime}}=V_{l_{1}}^{i^{\prime}}$, and the corresponding route is $\omega_{k_{1}}$; go to (5).

(5) When $A C_{k_{1}} \leq A C_{k_{2}}$, set $V_{l}^{i+1}=V_{l_{1}}^{i^{\prime}+1}, \Omega_{l_{2}}^{\prime}=\Omega_{l_{2}}^{\prime} \backslash V_{l_{2}}^{j^{\prime}}$, $\Omega_{l_{2}}^{\prime}=\Omega_{l_{2}}^{\prime} \backslash V_{l_{2}}^{j^{\prime}}, i=i+1$, and if $V_{l}^{i+1}$ is not a railway station, go to (3); otherwise, $n_{l}=n_{l}+1$; go to (6).

When $A C_{k_{1}}>A C_{k_{2}}$, set $V_{l}^{i+1}=V_{l_{2}}^{j^{\prime}+1}, \Omega_{l_{1}}^{\prime}=\Omega_{l_{1}}^{\prime} \backslash V_{l_{1}}^{i^{\prime}}$, $\Omega_{l_{2}}^{\prime}=\Omega_{l_{2}}^{\prime} \backslash V_{l_{2}}^{j^{\prime}}, i=i+1$, and if $V_{l}^{i+1}$ is not a railway station, go to (4); otherwise, $n_{l}=n_{l}+1$; go to (6).

(6) Delete those routes in $\Omega_{l_{1}}$ and $\Omega_{l_{2}}$ that do not satisfy constraint (5). If all the routes satisfy constraint (5) in $\Omega_{l_{1}}^{\prime}$ or $\Omega_{l_{2}}^{\prime}$, combine the two shortest routes in the corresponding parent; go to (2).

(7) Optimize the path structure of $n_{l}$ routes in $\Omega_{l}$, respectively.

Based on $\Omega_{l_{2}}$, another offspring can be generated in the same way.

Taking the following parents, the crossover operator that generates offspring 1 based on parent 1 is shown as follows:

\section{Parent 1: 1231146125712891013}

Parent 2: 4812521119312106713.

First, node 1 at the first locus in parent 1 is used as the node of offspring 1 at locus 1 . Then, gene segments $1-2$ in parent 1 and 1-9 in parent 2 are compared; $1-2$ are supposed to join offspring 1. Subsequently, 2-3 and 2-11 are compared. Offspring 1 is as below:

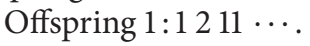


(3) Mutation Operator. A mutation operator with mutation probability $P_{c}$ is used to strengthen the global optimization ability of the GA. According to the types of genes randomly selected from one individual, exchange or insertion mutations are made: if the selected gene is a bus stop, it will be randomly inserted into another locus (insertion mutation); if the selected gene is a station, it will be randomly replaced by another station (exchange mutation). To guarantee the quality of the mutation, the acceptance probability of a mutation solution is

$$
p= \begin{cases}1, & \Delta Z<0 \\ e^{\left(-\Delta Z / Z_{0}\right)}, & \Delta Z \geq 0\end{cases}
$$

where $Z_{0}$ is the fitness of the best individual so far and $\Delta Z$ is the objective difference of the solution mutation before and after.

(4) Elite Preservation Strategy and Algorithm Termination Rules. To preserve the elite individuals of parent populations, the worst $4 \%$ of offspring individuals are replaced by the same proportion of the best ones. The termination rules of the GA make use of the maximum generations $T_{\max }$ or the maximum generations $T_{0}$ without improving the best solution so far.

\section{Numerical Examples}

The benchmark problem is taken from [6]. The network includes 55 bus stops and 4 railway stations, serving $2 *$ 2.5 square miles. The demand density of each stop per period (one hour) is 200 passengers. The values of the model parameters are shown in Table 1 . The GA is designed based on the C\# language. In the GA, we take $n=120, P_{c}=0.8, P_{m}=$ $0.08, T_{\max }=1200, T_{0}=100, \underline{\alpha}=0.05, \bar{\alpha}=0.30$, and $T_{\alpha}=$ 20 .

6.1. M-to-1 Demand Pattern. Railway station 56 is regarded as the central business district of the service area and the destination of all the passengers, so the demand is a distribution pattern between multiple stops and one station. The best feeder-bus network is shown in Figure 1 and Table 2.

Figure 2 shows a change in the objective function when the number of generations increases in the solving process. It illustrates that the GA presented in this paper performs a fast convergence speed.

6.2. Comparison of Best Solutions. To compare the optimal solutions with other approaches, we neglect the transfer cost (i.e., $\lambda_{j}=0$ ) and make the total cost of this paper accord with other methods, including saving heuristics [6], displacement heuristics, basic TS [14], and TS with intensification [16]. However, the bus riding-time cost is roughly approximated by estimating the total passenger-miles in these studies and there are some differences in constraints (8) and (9) in the models of $[6,16]$. Table 3 gives the best solutions of these studies.

The results show that the total cost of the GA saves $8.0 \%, 1.3 \%, 1.2 \%$, and $1.4 \%$ compared to the other approaches $[6,14,16]$, respectively. Because the length of each route is
TABLE 1: Model parameters.

\begin{tabular}{lcc}
\hline Parameter & Unit & Value \\
\hline$C$ & Seat & 50 \\
$\rho$ & & 1.2 \\
$t$ & Hour & 0.16 \\
$N$ & Vehicle & 110 \\
$\lambda_{w}$ & \$/passenger-hour & 8 \\
$\lambda_{r}$ & \$/passenger-hour & 4 \\
$\lambda_{T}$ & \$/passenger-hour & 4.5 \\
$\lambda_{i}$ & \$/passenger & 0.03 \\
$\lambda_{b}$ & \$/vehicle-mile & 3 \\
$V_{B}$ & Mile/hour & 20 \\
$V_{T}$ & Mile/hour & 30 \\
$\lambda$ & & 0.0001 \\
\hline
\end{tabular}

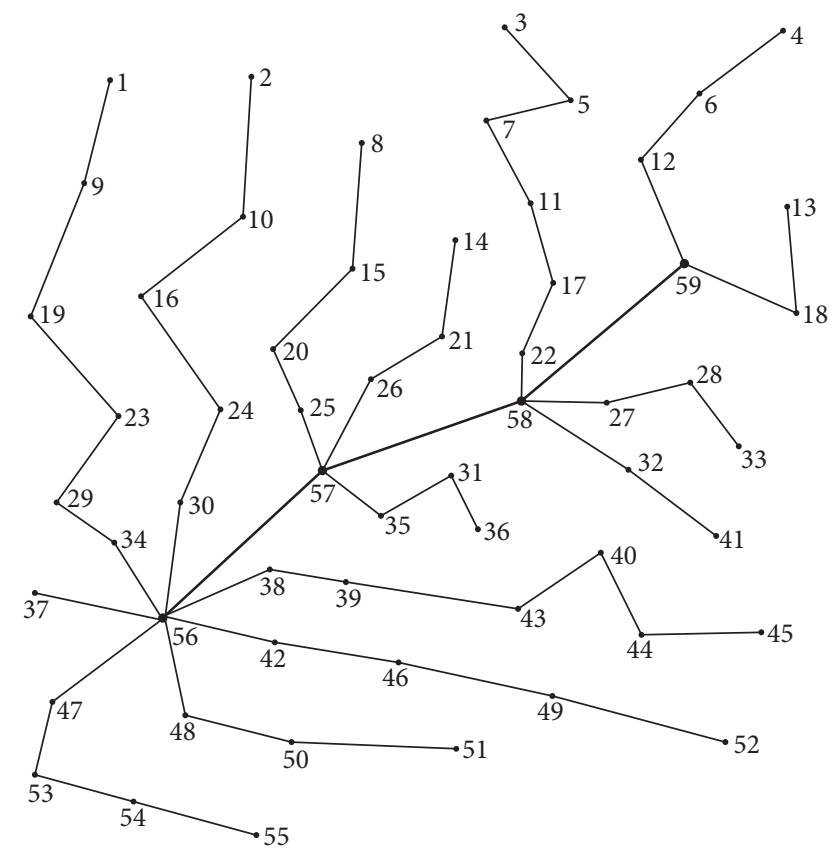

Figure 1: Optimal network under M-to-1.

TABLE 2: Solution indicators under M-to-1.

\begin{tabular}{lc}
\hline Total cost (\$) & 6474 \\
Number of routes & 15 \\
Average length per route (miles) & 1.02 \\
Average frequency per route (trips/hour) & 22.12 \\
\hline
\end{tabular}

not arbitrarily limited in our paper compared with the other studies, a better solution is obtained and the number of routes decreases. In the best solution of the GA, the number of routes and average route length are similar to those in the saving heuristics, while the total cost is lower.

6.3. M-to-M Demand Patterns. To study the change in the optimal network under various demand distributions, the 
TABLE 3: Comparison of the best solutions.

\begin{tabular}{lccccc}
\hline Solution approaches & Total cost (\$) & Route no. & $\begin{array}{c}\text { Average length per } \\
\text { route (miles) }\end{array}$ & $\begin{array}{c}\text { Average frequency per } \\
\text { route (trips/hour) }\end{array}$ & $\begin{array}{c}\text { Average computational } \\
\text { time (seconds) }\end{array}$ \\
\hline Saving heuristics [6] & 6824 & 16 & 1.05 & 21.06 & - \\
Displacement heuristics [14] & 6363 & 18 & 0.84 & 22.54 & - \\
Basic TS [14] & 6355 & 18 & 0.85 & 22.18 & - \\
TS with intensification [16] & 6371 & 19 & 0.80 & 22.76 & 13.8 \\
GA (in this paper) & 6281 & 15 & 1.01 & 22.01 & 21.6 \\
\hline
\end{tabular}

TABLE 4: Indicators of optimal solutions under various demand distributions.

\begin{tabular}{|c|c|c|c|c|c|c|c|c|c|c|}
\hline \multirow{2}{*}{$a$} & \multirow{2}{*}{ System cost ( $\$)$} & \multicolumn{4}{|c|}{ Station's transfer passengers } & \multirow{2}{*}{$\begin{array}{c}\text { Number of } \\
\text { routes }\end{array}$} & \multirow{2}{*}{$\begin{array}{c}\text { Average } \\
\text { route length }\end{array}$} & \multirow{2}{*}{$\begin{array}{c}\text { Average } \\
\text { route frequency }\end{array}$} & \multirow{2}{*}{$\begin{array}{l}\text { Average travel } \\
\text { time (bus : train) }\end{array}$} & \multirow{2}{*}{$\begin{array}{l}\text { Nonlinear } \\
\text { coefficient }\end{array}$} \\
\hline & & 56 & 57 & 58 & 59 & & & & & \\
\hline 50 & 6515 & 1600 & 3800 & 4600 & 1000 & 19 & 0.79 & 21.88 & $0.51: 0.53$ & 1.28 \\
\hline 40 & 6511 & 1400 & 3600 & 5000 & 1000 & 19 & 0.80 & 21.93 & $0.51: 0.51$ & 1.27 \\
\hline 30 & 6505 & 800 & 3800 & 4600 & 1800 & 18 & 0.84 & 22.06 & $0.50: 0.48$ & 1.27 \\
\hline 20 & 6494 & 1000 & 3000 & 5200 & 1800 & 18 & 0.84 & 21.99 & $0.50: 0.48$ & 1.25 \\
\hline 10 & 6476 & 800 & 2800 & 5600 & 1800 & 16 & 0.93 & 22.21 & $0.47: 0.41$ & 1.25 \\
\hline 0 & 6437 & 800 & 2800 & 4800 & 2600 & 17 & 0.89 & 22.04 & $0.50: 0.42$ & 1.22 \\
\hline
\end{tabular}

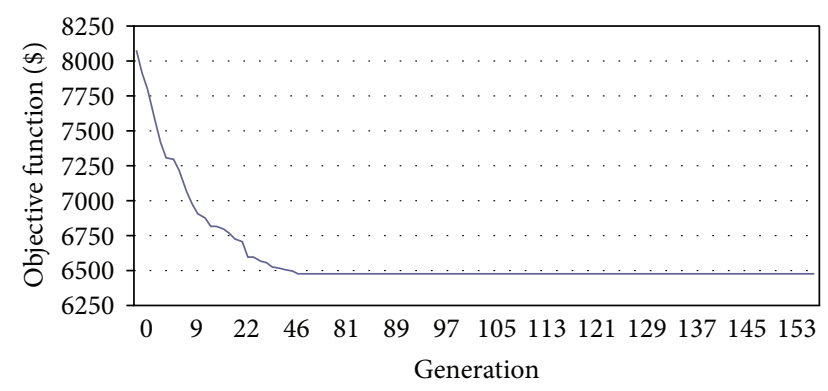

FIGURE 2: Convergence efficiency of GA under M-to-1.

patterns of passenger demand between each stop and 4 railway stations are generated by an arithmetic progression, the first term of which is $a$ and the common difference is $q$. For example, when $a=20$ and $q=20$, the number of passengers from each stop to stations $56-59$ is $20,40,60$ and 80 , respectively. Obviously, the imbalance of the demand distribution increases when $a$ decreases from 50 to 0 . The results under different values of $a$ are shown in Table 4 and Figure 3 shows the optimal feeder-bus network under the uniform distribution demand between the 4 railway stations $(q=0)$.

Figure 4 shows a change in the objective function under the demand pattern of M-to-M and $q=0$. It shows that the convergence speed of GA under M-to-M is satisfactory as well as M-to-1. From Table 4, the following observations regarding the demand distributions effect on the optimal feeder-bus networks can be found.

(1) For a given railway station, with the increase in passengers who terminate at a station, the number of passengers who choose to feed into this station increases grossly, as seen in Figure 5.

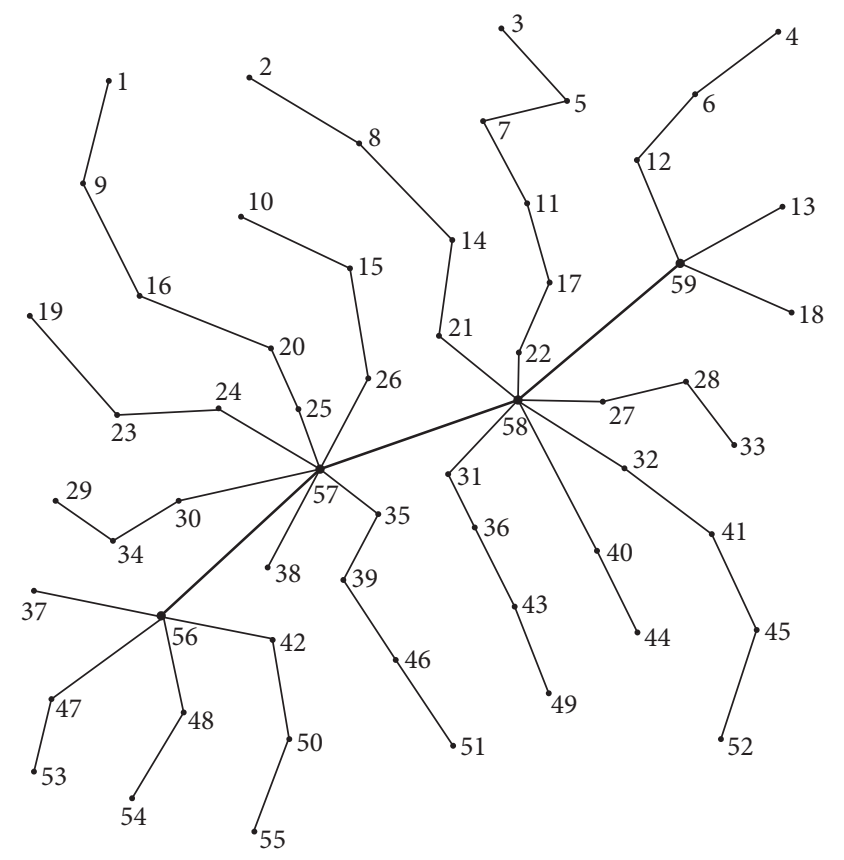

FIGURE 3: Optimal feeder-bus network under $q=0$.

(2) The demand distribution has an obvious effect on the average riding time by bus and train, as shown in Figure 6. With the increase in the demand imbalance between the stations, the difference in the average travel time in the two traffic modes becomes gradually more significant and the total travel time on the integrated transport network decreases simultaneously because the feeder station and route structure are influenced by major passengers. 


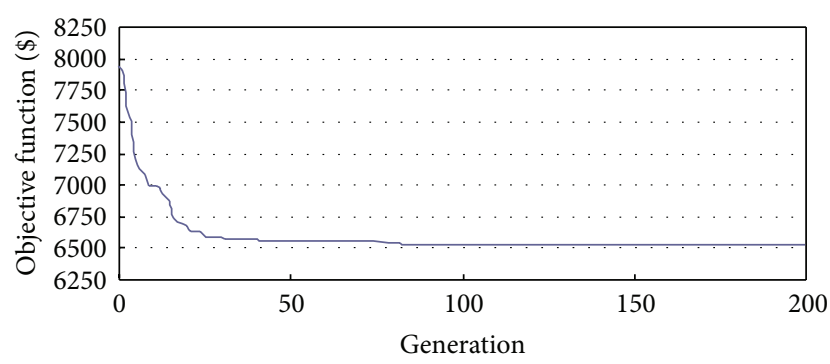

FIGURE 4: Convergence efficiency of GA under M-to-M.

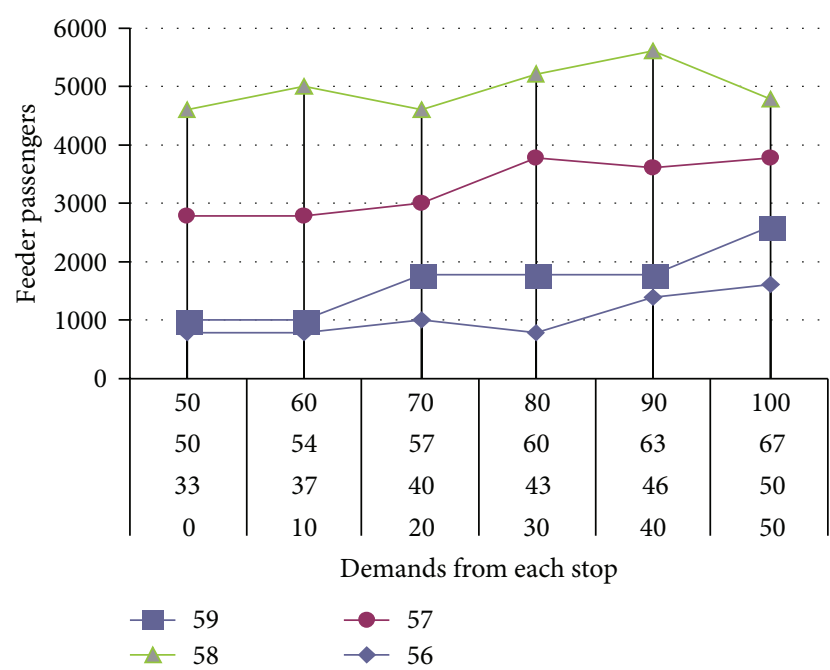

FIGURE 5: Effect of termination passengers on feeder passengers at stations.

(3) As Figure 7 shows, with the increase in the demand imbalance between stations, namely, the concentration of demand destinations, passengers' nonlinear coefficient falls and the system total cost also decreases remarkably. The reason is that the concentration of demand destinations makes most passengers obtain a better service, which causes a decline in the system's total cost.

According to the above analysis and the difference between Figures 1 and 3, we can draw the conclusion that the demand distribution has a great effect on the volume of passengers choosing given feeder-bus stations and the path structure of feeder routes, which will further influence the total cost of the whole feeder system.

\section{Conclusions}

This paper studies the optimal design problem of a feeder-bus network under the demand pattern of M-to-M. The drawback in most existing related research, that only a single destination exists (M-to-1 demand pattern), is modified to the Mto-M pattern for better accordance with the realistic demand distribution. In order to minimize the passenger travel cost and transit operating cost, an integrated public transport system of a feeder-bus network and railway is regarded as a
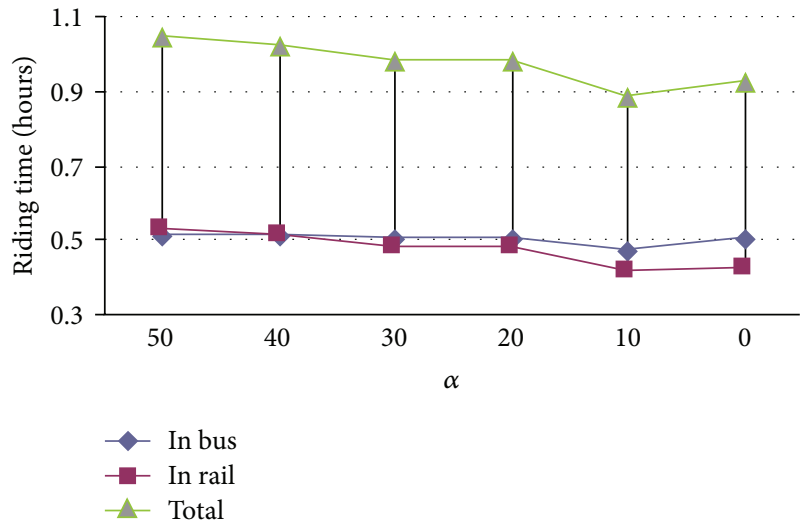

FIgURE 6: Effect of demand distribution on passengers' travel time.

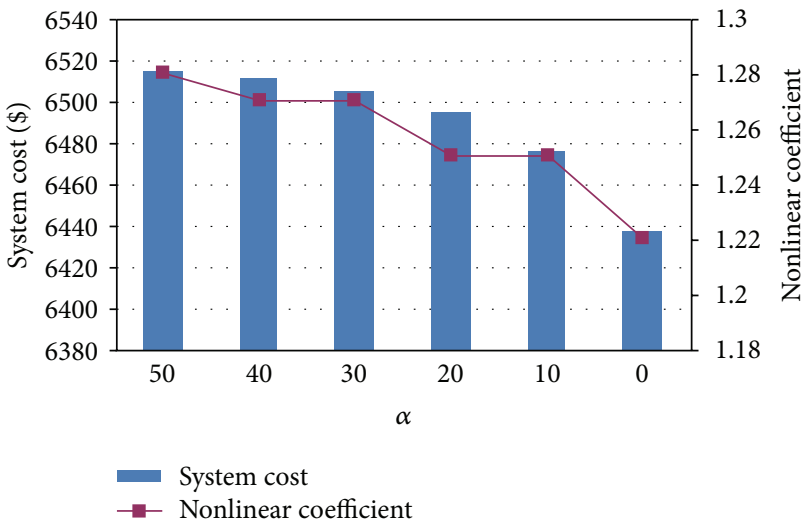

FIGURE 7: Relation between demand distribution and system cost or nonlinear coefficient.

whole to calculate the passenger travel cost overall. The results show that passenger demand distributions have a significant influence on feeder-bus network construction, especially on the feeder stations, the paths and frequencies, of feeder bus routes. Therefore, demand distributions should be considered when designing a feeder-bus network.

Usually public transportation network planning has symmetry, though differences in two directions are not considered in this paper. If the demand on a feeder-bus network has an obvious tidal phenomenon with time distribution, and the operating frequencies in different directions differ greatly, a directed feeder-bus network should be designed according to the directional demand.

One prerequisite of the FBNDP in this paper is that the station layout has to be determined. In our work, the optimal frequencies of feeder bus routes are determined according to the feeder passenger between rail and bus transit system. If one feeder-bus route only services few passengers, it could be removed from the feeder-bus network.

\section{Acknowledgments}

This research is supported by the National Natural Science Foundation of China (70901076, 71171200), the Science and 
Technology Research Development Program of China Railway Corporation (Major Program, 2013X004-A), and the Research Fund for Fok Ying Tung Education Foundation of Hong Kong (Project no. 132017).

\section{References}

[1] A. D. May, "Integrated transport strategies: a new approach to urban transport policy formulation in the UK," Transport Reviews, vol. 11, no. 3, pp. 223-247, 1991.

[2] R. M. Stanger and V. R. Vuchic, "The design of bus-rail transit facilities," Transit Journal, vol. 5, no. 4, pp. 61-72, 1979.

[3] J. A. Dunn Jr., "Coordination of urban transit services: the german model," Transportation, vol. 9, no. 1, pp. 33-43, 1980.

[4] B. F. Byrne and V. Vuchic, "Public transportation line positions and headways for minimum cost," Traffic Flow and Transportation, pp. 347-360, 1972.

[5] G. K. Kuah and J. Perl, "Optimization of feeder bus routes and bus-stop spacing," Journal of Transportation Engineering, vol. 114, no. 3, pp. 341-354, 1988.

[6] G. K. Kuah and J. Perl, "The feeder-bus network-design problem," Journal of the Operational Research Society, vol. 40, pp. 751-767, 1989.

[7] B. F. Byrne, "Cost minimizing positions, lengths and headways for parallel public transit lines having different speeds," Transportation Research, vol. 10, no. 3, pp. 209-214, 1976.

[8] V. F. Hurdle, "Minimum cost locations for parallel public transit lines," Transport Science, vol. 7, no. 4, pp. 340-350, 1973.

[9] S. C. Wirasinghe, V. F. Hurdle, and G. Newell, "Optimal parameters for a coordinated rail and bus transit system," Transportation Science, vol. 11, no. 4, pp. 359-374, 1977.

[10] S. C. Wirasinghe, "Nearly optimal parameters for a rail/feederbus system on a rectangular grid," Transportation Research A, vol. 14, no. 1, pp. 33-40, 1980.

[11] S. Chien and P. Schonfeld, "Joint optimization of a rail transit line and its feeder bus system," Journal of Advanced Transportation, vol. 32, no. 3, pp. 253-284, 1998.

[12] S. Chien and Z. Yang, "Optimal feeder bus routes on irregular street networks," Journal of Advanced Transportation, vol. 34, no. 2, pp. 213-248, 2000.

[13] S. Chien, Z. Yang, and E. Hou, "Genetic algorithm approach for transit route planning and design," Journal of Transportation Engineering, vol. 127, no. 3, pp. 200-207, 2001.

[14] C. L. Martins and M. V. Pato, "Search strategies for the feeder bus network design problem," European Journal of Operational Research, vol. 106, no. 2-3, pp. 425-440, 1998.

[15] P. Shrivastav and S. L. Dhingra, "Development of feeder routes for suburban railway stations using heuristic approach," Journal of Transportation Engineering, vol. 127, no. 4, pp. 334-341, 2001.

[16] S. N. Kuan, H. L. Ong, and K. M. Ng, "Applying metaheuristics to feeder bus network design problem," Asia-Pacific Journal of Operational Research, vol. 21, no. 4, pp. 543-560, 2004.

[17] S. N. Kuan, H. L. Ong, and K. M. Ng, "Solving the feeder bus network design problem by genetic algorithms and ant colony optimization," Advances in Engineering Software, vol. 37, no. 6, pp. 351-359, 2006.

[18] F. Ciaffi, E. Cipriani, and M. Petrelli, "Feeder bus network design problem: a new metaheuristic procedure and real size applications," Procedia-Social and Behavioral Sciences, vol. 54, pp. 798807, 2012. 


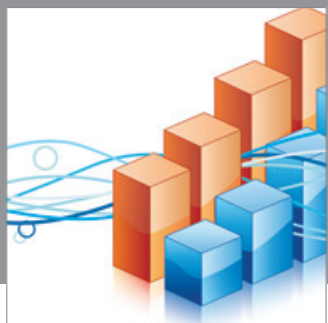

Advances in

Operations Research

mansans

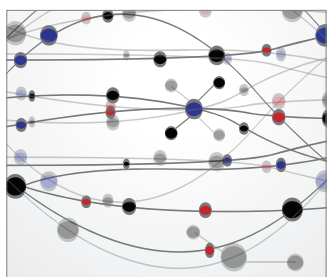

The Scientific World Journal
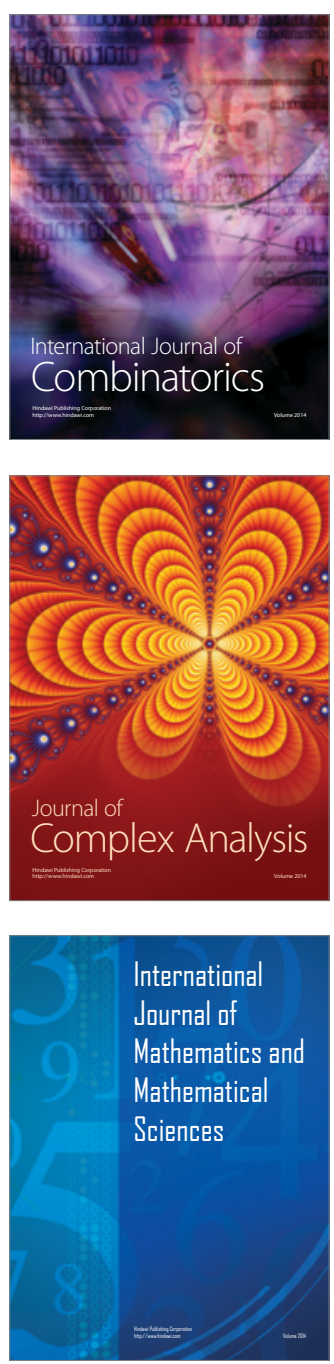
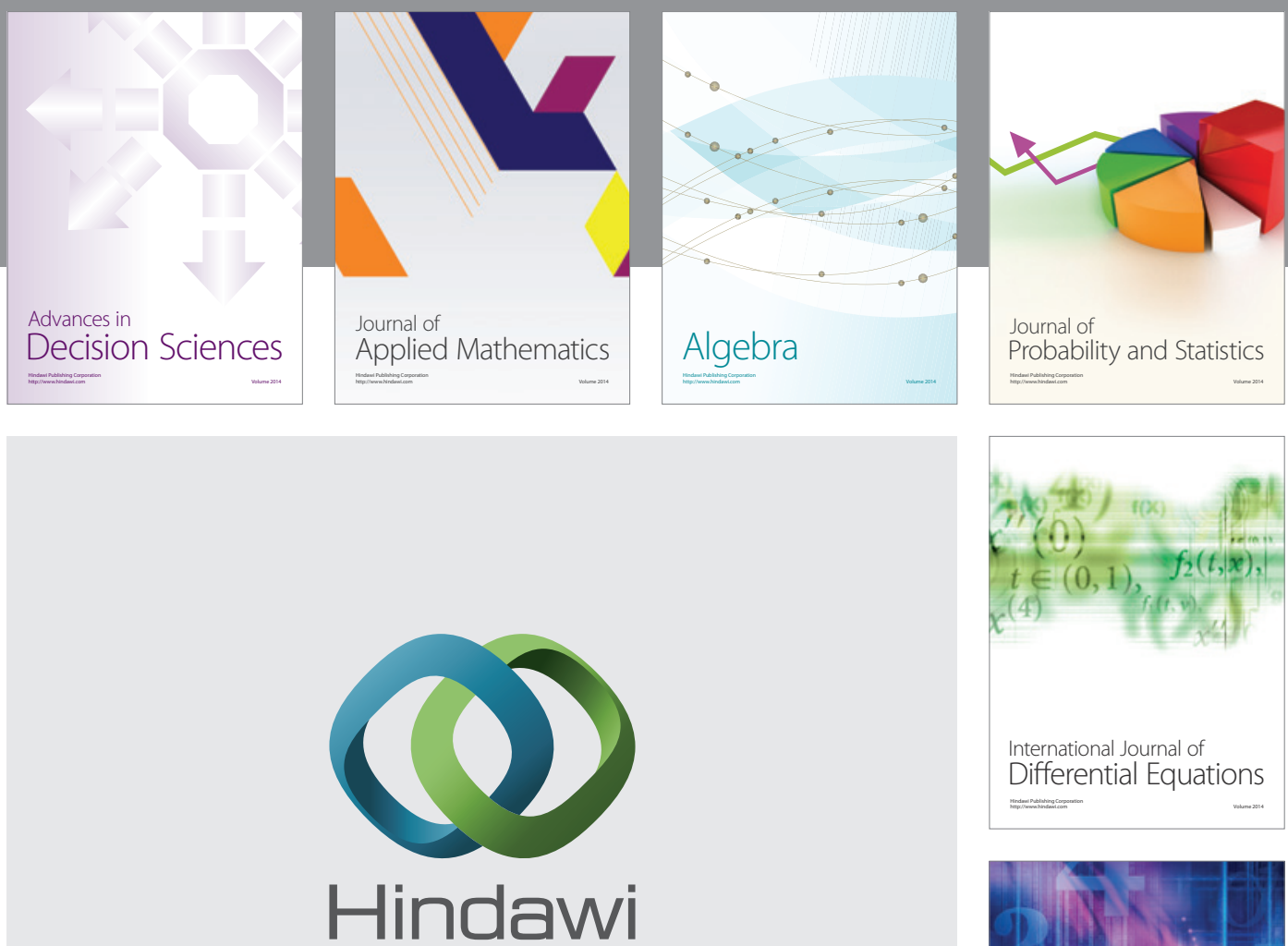

Submit your manuscripts at http://www.hindawi.com
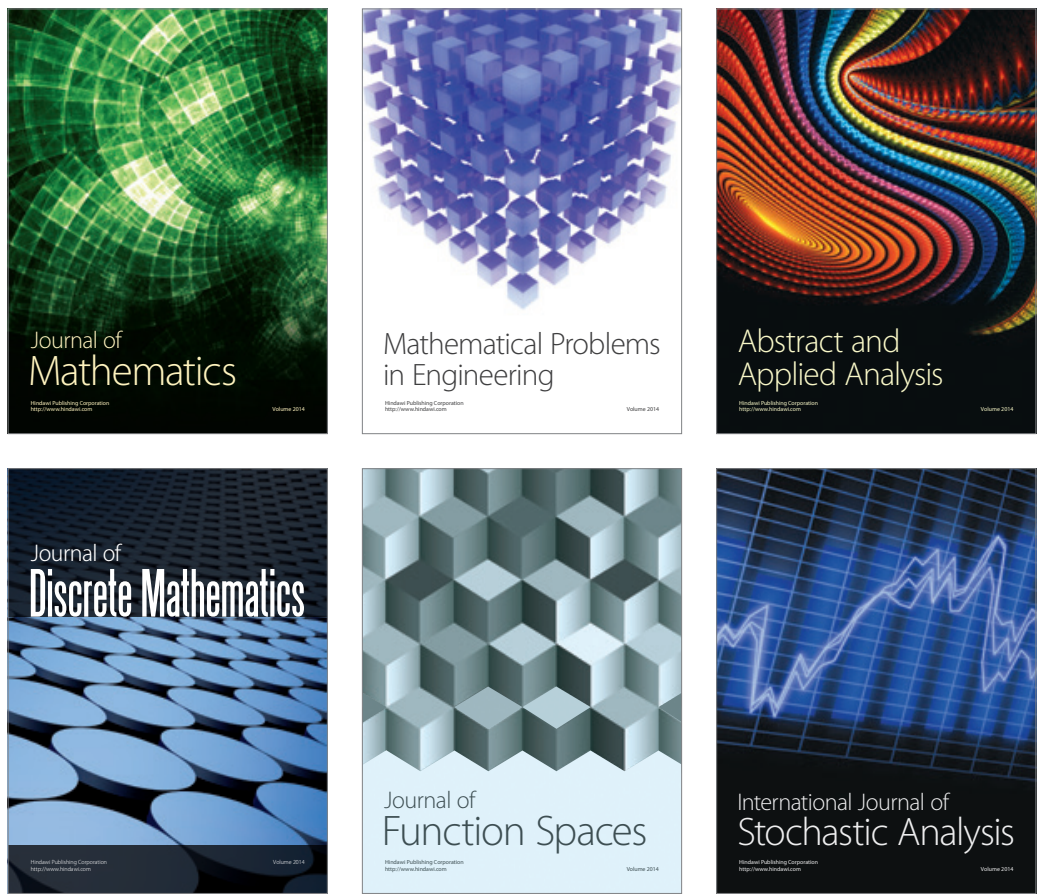

Journal of

Function Spaces

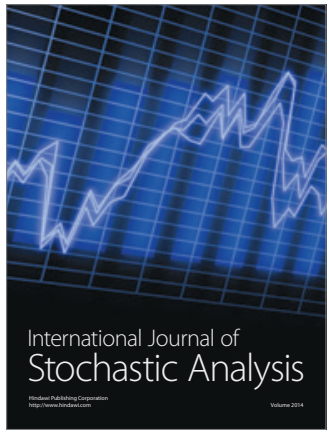

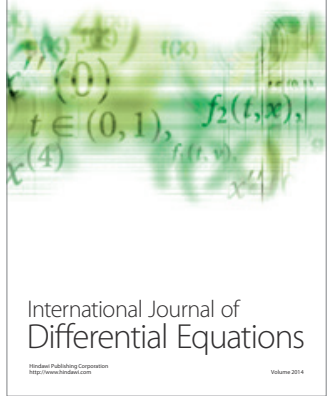
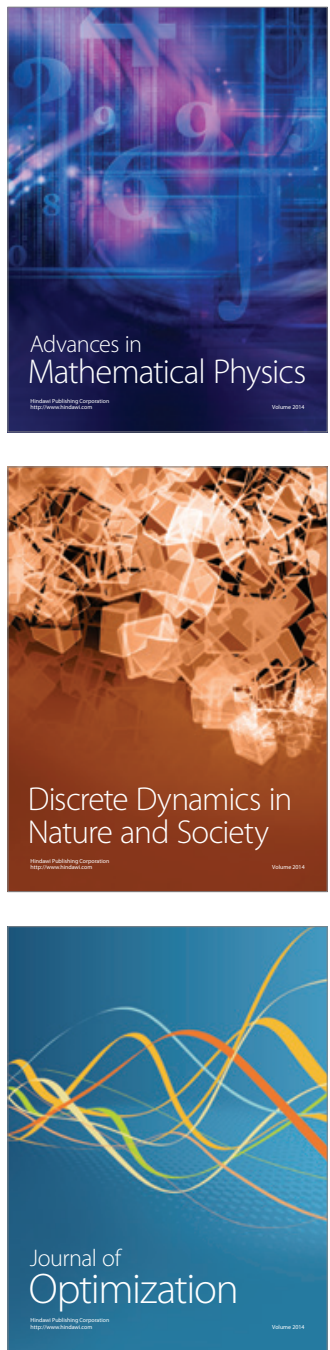